\title{
Correspondence
}

\begin{abstract}
Consultant responsibility
DeAr Sirs

I should like, through your columns, to draw upon the experience and opinion of College members in respect of an aspect of consultant responsibility which appears at least as problematical as participation in a multidisciplinary team.

It appears now to be common practice in psychiatric hospitals for a single nominated consultant to be associated with the ward or wards designated for disturbed or especially difficult patients. This arrangement is likely to apply at least at the level of 'clinical administration' of the ward (i.e. determining policies and monitoring their implementation); it will perhaps also extend to the clinical management of the individual patients throughout the period of their stay on the ward. There are sound reasons in favour of such a scheme-it facilitates the establishment of a unified ward team who can evolve and pursue a consistent therapeutic philosophy for all patients; ward activities are not interrupted by regular (or irregular) visits by numerous consultants; junior doctors appointed to the ward for training experience are responsible to a single consultant. For individual patients there will, of course, be some discontinuity of medical supervision in addition to the other changes inherent in the transfer to the 'disturbed' environment; it is likely, however, that consultants will have satisfied themselves that proper consultation on treatment plans takes place at the time of admission and at appropriate stages throughout the patients' stay, so that the management in the ward is consistent with the long-term treatment plan.
\end{abstract}

In practice, arrangements of this sort can work very well. Problems arise, however, when the question of consultant responsibility is addressed, particularly in relation to detained patients for whom the concept of a Responsible Medical Officer (RMO) applies. To what extent can the original consultant be held responsible while a colleague is making day-to-day decisions (such as leave arrangements or modifying medication regimes)? I understand that in some units the patient is formally re-allocated to the ward consultant for the duration of the stay, even if this is only for a day or two; is this practice not inconsistent with the spirit of the Act (which emphasises a comprehensive and continuous treatment plan) by distancing the patient even further from the clinical influence of the Consultant who will be responsible for providing care and treatment on a long term basis?

The essence of the dilemma appears to be this: can a consultant, who undertakes full responsibility for the clinical care of his patient and is professionally accountable to the Courts and the GMC, agree to share his responsibility with a colleague who has attained equal status? And if not, how can the best interests of patients and ward staff be served, in accordance also with the recommendations of the supervising authorities (ENB and JCHPT) who will not give educational approval to units where several consultants manage their patients independently?

I would be interested to hear the views of the College and of your readers on this difficult issue.

L. M. KREMER

Cherry Knowle Hospital
Ryhope, Sunderland

\section{Listening to the relatives!}

\section{DeAR Sirs}

Interviews with patients' relatives are part of our everyday work and are important in terms of diagnosis, treatment, discharge and prevention of relapse. Yet in these days of audit, when most of our items of work are counted and compared to an ideal average, interviews, which are time-consuming and good for consumer satisfaction, are seldom recorded. How many, with whom, where, why...? To answer these questions for an average consultant psychiatrist working in a district general hospital psychiatric unit a year's prospective study was made.

There were $\mathbf{7 3 0}$ interviews with $\mathbf{4 0 2}$ true relatives, aged over 16, of 277 patients. The relative had asked for the interview in 288 cases, the doctor in 266, and 176 were jointly arranged, e.g. repeat interviews. Twenty-five types of relative were seen. Female relatives came more frequently than male; more mothers, daughters, sisters, aunts, nieces etc. than the male counterparts. Were men less concerned, at work, or in the pub? Husbands, as relatives, did outnumber wives, but this is to be expected as there were twice as many female patients as male.

A hospital out-patients' clinic was the setting for 292 interviews, 147 interviews were held in patients' own homes and 89 relatives were seen on various wards. The longest and most intense interviews, 191, were held during a weekly 'relatives' clinic'. The remaining 11 were in diverse settings. The patient was present during $75 \%$ of the interviews. In almost half the interviews, information was gathered which was of value in reaching or confirming the diagnosis. The diagnoses were, in general terms, affective disorder 102 , paranoid illness 49, neurotic illness 45 , dementia 35 , alcohol problem 24, others 22 (less than five of each). Surprisingly the relative sought a diagnosis in only $22 \%$ of interviews and the trend was that the more 'psychotic' the diagnosis the less the question was asked. In $83 \%$ of interviews joint management and treatment plans were discussed. These included admission, day hospital, clinic appointment, community nurse visits, medication, referral to psychologist, occupational therapist, physiotherapist, speech therapist and, surprisingly often, dietician. Trial periods of leave were arranged and evaluated. In $60 \%$ of interviews other 